\title{
Effect of Asynchronous Transfer Mode Adaptation Layers and Service Class on Network Performance
}

\author{
Michael Asante \\ Department of Computer Science \\ Kwame Nkrumah University of \\ Science and Technology
}

\author{
Francis Kwadzo Agbenyegah \\ Ghana Communication Technology \\ University \\ Department of Computer Science \\ Kwame Nkrumah University of \\ Science and Technology
}

\author{
James Owusu - Kusi \\ Department of Computer Science \\ Kwame Nkrumah University of \\ Science and Technology
}

\begin{abstract}
This research work investigated the effect of Asynchronous Transfer Mode Adaptation Layers and Service Classes on the performance of a network. The relation between applications such as Voice, Ftp and Email, Asynchronous Transfer Mode (ATM) Adaptation Layers and ATM Service Classes and their accompanying effect on the performance of a network was investigated. Emphasis on the relation between the ATM Adaptation Layers and ATM Service Classes was centered on Email Download and Upload Response Time, Ftp Download and Upload Response Time, Voice Packet Delay Variation and Voice Packet End to End Delay.
\end{abstract}

Two Scenarios were evaluated through simulations using Riverbed Modeler Academic Edition version 17.5. The results show that the type of ATM Adaptation Layer and Service Classes used for an application has an effect on the performance of a network.

\section{General Terms}

ATM Adaptation Layers and ATM Service Classes

\section{Keywords}

Email Download and Upload Response Time, Ftp Download and Upload Response Time, Voice Packet Delay Variation and Voice Packet End to End Delay

\section{INTRODUCTION}

Computer networks has become an indispensable element of our day-to-day operations. Newer network technologies are sought after from time to time to meet the ever-increasing need of high bandwidth and low delay over long distances. To satisfy these needs, several high-speed network techniques have been developed. Networks are normally developed based on the type of data to be transported. Thus, circuit-switched networks, which included the public switched telephone network and high-speed digital transmission facilities, were primarily used to transport delay-sensitive information, such as voice and video. In comparison, on packet-based networks, such as X.25 and Frame Relay, information can tolerate a degree of delay. Network users can select a networking technology to satisfy a specific communications application, but most organizations support a mixture of applications. Thus, most organizations are forced to operate multiple networks, resulting in a degree of inefficiency and escalating communications costs.

This inefficiency on the part of early network technologies to encapsulate different applications such as voice, video and data together in a single network brought to fore the creation of a new network technology by name (ATM) which by combining the features from other technologies, ATM enables a single network to support voice, data, and video.

\section{PROBLEM STATEMENT}

Although each ATM Adaptation Layer is optimized for a specific type of traffic, there is no stipulation in the standards that ATM Adaptation Layers (AALs) designed for one class of traffic cannot be used for another. In fact, many vendors of ATM equipment currently manufacture products that use AAL5 to support all the above classes of traffic, and most activities at the ATM Forum have focused on AAL5 [11]. There is limited literature on the effect of the various types of Adaptation Layers and the applications that they transport on the performance of a network. This paper therefore investigated the effect of the various types of adaptation layers and the applications they transport and their resultant effect on network performance

\section{RESEARCH OBJECTIVES}

The objective of this research work is to examine the effect of ATM Adaptation layers and Service Classes on network performance. The specific objectives of this research work are to:

i. Examine the effect of ATM Adaptation layers and Service Classes in the transmission of Voice Application over a network.

ii. Examine the effect of ATM Adaptation layers and Service Classes in the transmission of an Email document over a network.

iii. Examine the effect of ATM Adaptation layers and Service Classes in the transmission of an FTP file over a network.

\subsection{Research Questions}

The experimental setup was done to answer the following research questions

RQ1: What is the effect of ATM Adaptation layers and Service Classes in the transmission of a Voice application over a network?

RQ2: What is the effect of ATM Adaptation layers and Service Classes in the transmission of an Email document over a network? RQ3: What is the effect of ATM Adaptation layers and Service Classes in the transmission of an FTP file over a network? 


\section{METHODOLOGY}

The main aim of this research work is to examine the effect of ATM Adaptation layers and Service Classes on network performance. An evaluation of the performance of the network under two (2) different scenarios were conducted where two Adaptation Layers (AAL2 and AAL5) and two Services (Constant Bit-Rate (CBR) and Unspecified Bi-Rate (UBR)) were used. The simulation was done for 20 work stations and simulated in a way that all the 20 work stations would access FTP, Email and Voice application under the two different scenarios. Each scenario for the applications (voice, Ftp and Email) under study was ran five times using 100, 300, 500,700 and 1000 statistical values for ten (10) minutes. The five statistical values would help in appreciating the behavior of a network during low traffic transmission (100 - 300), medium traffic transmission (500) and high traffic transmission $(700$ - 1000). Five data points were calculated as the average for all the five (5) quanta of statistics picked. An average of all the five average quantum statistics were generated to be representative of the behavior of the network at low, medium and high traffics.

The following performance metrics were used for performance evaluation under the two scenarios:
i. $\quad$ Email Download Response Time $(\mathrm{Sec})$
ii. $\quad$ Email Upload Response Time (Sec)
iii. $\quad$ FTP Download Response Time (Sec)
iv. $\quad$ FTP Upload Response Time (Sec)
v. Packet Delay Variation
vi. Packet End-to-End Delay (Sec)

\section{RESULTS AND DISCUSSION}

This session discusses the simulation result after running the two scenarios for $100,300,500,700$ and 1000 values; the average of the each of five simulations runs were calculated. The two scenarios presented were;

i)

CBR-UBR (Scenario making use of both Constant Bitrate and Unspecified Bitrate as Service Classes and AAL2 and AAL5 as ATM Adaptation Layers for the applications.)

ii) UBR-UBR (Scenario making use of only Unspecified Bitrate as Service Class for all the applications and making use of AAL5 accordingly as ATM Adaptation layers.)

\subsection{Results for Email Download Response Time} (Sec)

The first scenario (CBR-UBR) produced an average Email Download Response Time of 0.84543 and the second scenario (UBR-UBR) produced an average Email Download Response Time of 0.758332 as shown in table 1 . The Time elapsed between sending request for emails and receiving emails from the email server in the network in the first scenario is higher than that of the second scenario.
Table 1: Email Download Response

\begin{tabular}{|l|c|c|c|c|c|c|}
\hline \multirow{2}{*}{ SCENARIOS } & \multicolumn{5}{|c|}{ VALUES PER STATISTIC } & \multirow{2}{*}{ AVERAGE } \\
\cline { 2 - 6 } & 100 & 300 & 500 & 700 & 1000 & \\
\hline CBR_UBR & & & & & & \\
(Sec) & 0.872105 & 0.844192 & 0.84421 & 0.822511 & 0.844133 & 0.84543 \\
\hline UBR_UBR & & & & & & \\
(Sec) & 0.7572 & 0.753265 & 0.758953 & 0.758873 & 0.763368 & 0.758332 \\
\hline
\end{tabular}

In the first Scenario (CBR_UBR), the adaptation layer and services in the Data Station for Email were assigned as follows in table 2: A higher Email Download Respond Time and for that matter poor respond time was recorded because, Emails do not make use of AAL2 for its transport. In this scenario, AAL2 assigned for voice had taken part of the transport channel and the Email application was forced to make use of its transport channel and that of Ftp (AAL5).

\begin{tabular}{|c|c|c|c|}
\hline APPLICATION & $\begin{array}{l}\text { ATM ADAPTATION } \\
\text { LAYER }\end{array}$ & $\begin{array}{l}\text { SERVICE } \\
\text { CLASS }\end{array}$ & OUTPUT \\
\hline Voice & AAL2 & UBR & \multirow{3}{*}{0.84543} \\
\hline Ftp & AAL5 & UBR & \\
\hline Email & AAL 5 & UBR & \\
\hline
\end{tabular}

In the Second Scenario (UBR_UBR), the adaptation layer and services in the Data Station for Email were assigned as shown in table 3:

A lower Email Download Respond Time and for that matter a better response time was recorded because, Emails make use of AAL5 for its transport. The outputs of the two scenarios (bitrates against average response time were plotted as shown in figure1. From the graph, the Email had a wider spectrum of transport channel for it to be transported and also it can be shown that, the email download response time is much lower in the second scenario as compared to the first scenario.

Table3: Data Station for Emails

\begin{tabular}{|l|l|l|l|}
\hline APPLICATION & $\begin{array}{l}\text { ATM ADAPTATION } \\
\text { LAYER }\end{array}$ & $\begin{array}{l}\text { SERVICE } \\
\text { CLASS }\end{array}$ & \multirow{2}{*}{ OUTPUT } \\
\cline { 1 - 3 } Voice & AAL5 & UBR & \multirow{2}{*}{$\mathbf{0 . 7 5 8 3 3 2}$} \\
\cline { 1 - 3 } Ftp & AAL5 & UBR & \\
\cline { 1 - 3 } Email & AAL5 & UBR & \\
\hline
\end{tabular}




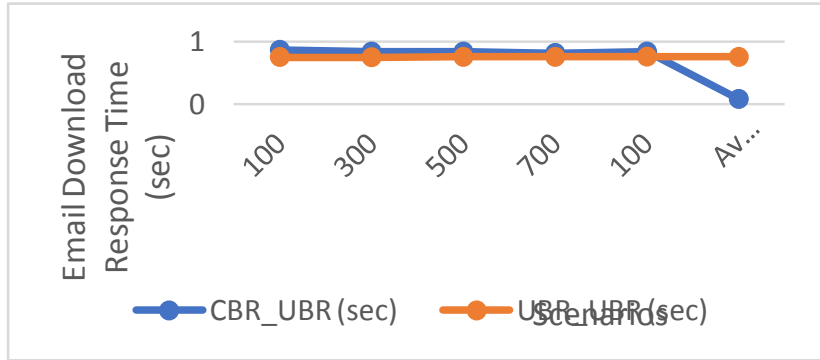

Fig. 1 Email download response time

5.2 Results for Email Upload Response Time (Sec) In the first scenario (CBR-UBR) produced an average Email Upload Response Time of 0.737016 whiles the second scenario (UBR-UBR) produced an average Email Upload Response Time of 0.71306. As seen in table 4, time elapsed between sending emails to the email server and receiving acknowledgments from the email server in the first scenario is higher than that of the second scenario

Table 4: Email Upload Response time

\begin{tabular}{|c|c|c|c|c|c|c|}
\hline \multirow{2}{*}{ SCENARIOS } & \multicolumn{5}{|c|}{ VALUES PER STATISTIC } & \multirow{2}{*}{ AVERAGE } \\
\cline { 2 - 6 } & 100 & 300 & 500 & 700 & 1000 & \\
\hline CBR_UBR (Sec) & & & & & & \\
& 0.723111 & 0.735267685 & 0.739442 & 0.740089 & 0.743169 & 0.737016 \\
\hline UBR_UBR (Sec) & & & & & & \\
& 0.727813 & 0.710648314 & 0.709214 & 0.709504 & 0.708121 & 0.71306 \\
\hline
\end{tabular}

In the first Scenario (CBR_UBR), the adaptation layer and services in the Data Station for Email were assigned as follows:

Table 5: Email Upload Response Time for Scenario 1

\begin{tabular}{|l|l|l|l|}
\hline APPLICATION & $\begin{array}{l}\text { ATM } \\
\text { ADAPTATION } \\
\text { LAYER }\end{array}$ & $\begin{array}{l}\text { SERVICE } \\
\text { CLASS }\end{array}$ & OUTPUT \\
\hline Voice & AAL2 & UBR & \multirow{2}{*}{0.737016} \\
\cline { 1 - 3 } Ftp & AAL5 & UBR & \\
\cline { 1 - 3 } Email & AAL5 & UBR & \\
\hline
\end{tabular}

A higher Email Upload Response Time and for that matter poor response time was recorded because, Emails do not make use of AAL2 for its transport. In this scenario, AAL2 assigned for voice had taken part of the transport channel and the Email application was forced to make use of its transport channel and that of Ftp (AAL5).

In the Second Scenario (UBR_UBR), the adaptation layer and services in the Data Station for Email were assigned as follows:
Table 6: Email Upload Response Time for Scenario 2

\begin{tabular}{|l|l|l|l|}
\hline APPLICATION & $\begin{array}{l}\text { ATM ADAPTATION } \\
\text { LAYER }\end{array}$ & $\begin{array}{l}\text { SERVICE } \\
\text { CLASS }\end{array}$ & \multirow{2}{*}{ OUTPUT } \\
\cline { 1 - 3 } Voice & AAL5 & UBR & \multirow{2}{*}{0.71306} \\
\cline { 1 - 3 } Ftp & AAL5 & UBR & \\
\hline Email & AAL5 & UBR & \\
\hline
\end{tabular}

A lower Email Upload Respond Time and for that matter a better response time was recorded because, Emails make use of AAL5 for its transport and the Service Class, UBR gives it a quality of service. In this scenario, the Email had a wider spectrum of transport channel for it to be transported since it took advantage of the adaptation layer of voice (AAL5), Ftp (AAL5) and its own. It also made use of the Service Class of Voice (UBR) and that of Ftp (UBR) thereby lowering the time spent in uploading the Email file. It can therefore be concluded that AAL5 adaptation layer and the UBR Service Class is good for Data such as Emails. Figure 2 shows the two scenarios when the simulation was run.

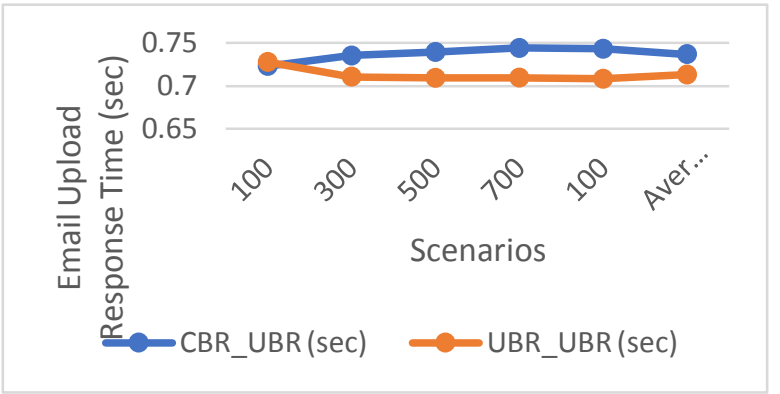

Figure 2: Email Upload Response Time.

\subsection{Results for FTP Download Response Time}

(Sec)

An average value of 1.133026 seconds and 1.144771 seconds were recorded respectively for CBR_UBR and UBR_UBR scenarios shown in table 7. The Time elapsed between sending a request and receiving the response packet in the first scenario is lower than that of the second Scenario.

In the first Scenario (CBR_UBR), the adaptation layer and services in the Data Station for Ftp were assigned as follows in table 8: A lower Download Response Time and for that matter a better response time was recorded in this scenario for Ftp file such as video because, AAL2 which is good for audio takes care of the audio aspect of the Ftp file while AAL5 which is good for data files takes care of the data aspect of the Ftp file. It also made use of the Service Class of Voice (UBR) and that of Ftp (UBR) there by lowering the time spend in downloading the Ftp file. 
Table7. FTP Download Response Time

\begin{tabular}{|c|c|c|c|c|c|c|}
\hline \multirow{2}{*}{ SCENARIOS } & \multicolumn{5}{|c|}{ VALUES PER STATISTIC } & \multirow{2}{*}{ AVERAGE } \\
\cline { 2 - 6 } & 100 & 300 & 500 & 700 & 1000 & \\
\hline CBR_UBR (Sec) & 1.117958 & 1.136719 & 1.136733 & 1.13682 & 1.136902 & 1.133026 \\
& & & & & & \\
\hline UBR_UBR (Sec) & 1.140569 & 1.139783 & 1.13986 & 1.163139 & 1.140505 & 1.144771 \\
& & & & & & \\
\hline
\end{tabular}

Table 8: FTP Download Response Time for Scenario 1

\begin{tabular}{|l|l|l|l|}
\hline APPLICATION & $\begin{array}{l}\text { ATM ADAPTATION } \\
\text { LAYER }\end{array}$ & $\begin{array}{l}\text { SERVICE } \\
\text { CLASS }\end{array}$ & \multirow{2}{*}{ OUTPUT } \\
\cline { 1 - 3 } Voice & AAL2 & UBR & \multirow{2}{*}{$\mathbf{1 . 1 3 3 0 2 6}$} \\
\cline { 1 - 3 } Ftp & AAL5 & UBR & \\
\hline Email & AAL5 & UBR & \\
\hline
\end{tabular}

In the second Scenario (UBR_UBR), the adaptation layer and services in the Data Station for Ftp were assigned as follows in table 9: A higher Ftp Download Response Time and for that matter poor response time was recorded because, AAL5 adaptation layer was assigned to the voice aspect of the Ftp file though the data aspect of the Ftp file took advantage of the adaptation layer of Email (AAL5) and its own. It could be deduced that; the voice aspect of the Ftp file was delayed which in turn affected the overall download response time for the Ftp File. It can therefore be concluded that, in downloading an Ftp File, two different adaptation layers (AAL2 and AAL5) are required. The AAL2 transports the voice aspect and AAL5 to transports the data aspect. It can also be deduced that UBR as a Service Class is better in downloading Ftp Files.

The figure 3 shows the result of the two scenarios when the simulation was run for 10 minutes. When the simulation was run for low traffic (100), the first scenarios (CBR_UBR) records a value of $1.11 \mathrm{sec}$ as against $1.14 \mathrm{sec}$. With a medium traffic, both records almost the same value of $1.14 \mathrm{sec}$., but when the traffic was high (700), the second scenarios (UBR_UBR) recorded a higher response time of $1.16 \mathrm{sec}$ against $1.13 \mathrm{sec}$ for CBR_UBR scenarios.

Table 9: FTP Download Response Time for Scenario 2

\begin{tabular}{|l|l|l|l|}
\hline APPLICATION & $\begin{array}{l}\text { ATM ADAPTATION } \\
\text { LAYER }\end{array}$ & $\begin{array}{l}\text { SERVICE } \\
\text { CLASS }\end{array}$ & \multirow{2}{*}{1.144771} \\
\cline { 1 - 3 } Voice & AAL5 & UBR & \\
\cline { 1 - 3 } Ftp & AAL5 & UBR & \\
\cline { 1 - 3 } Email & AAL5 & UBR & \\
\cline { 1 - 3 } & & &
\end{tabular}

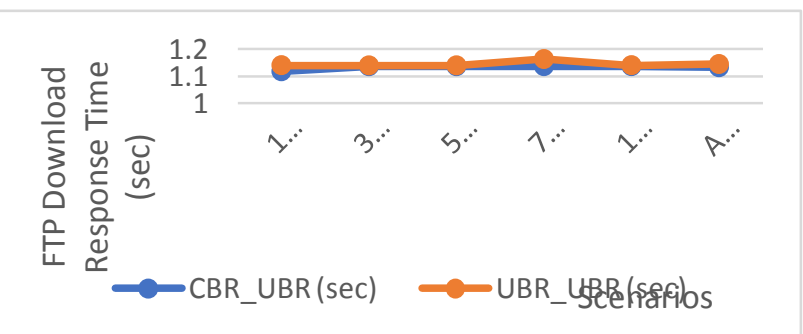

Figure 3: FTP Download Response Time
5.4 Results for FTP Upload Response Time ( $\mathrm{Sec}$ )

An average value of 1.129103 seconds and 1.135638 seconds were recorded respectively for CBR_UBR and UBR_UBR scenarios. The time elapsed between sending a file and receiving the response from a server in the first scenario is lower than that of the second Scenario as shown in table 10.

Table 10: FTP Upload Response Time

\begin{tabular}{|l|c|c|c|c|c|c|}
\hline \multirow{2}{*}{ SCENARIOS } & \multicolumn{5}{|c|}{ VALUES PER STATISTIC } & \multirow{2}{*}{ AVERAGE } \\
\cline { 2 - 6 } & 100 & 300 & 500 & 700 & 1000 & \\
\hline $\begin{array}{l}\text { CBR_UBR } \\
\text { (Sec) }\end{array}$ & 1.115002 & 1.132577 & 1.132621 & 1.13264 & 1.132676 & 1.129103 \\
\hline $\begin{array}{l}\text { UBR_UBR } \\
\text { (Sec) }\end{array}$ & 1.135511 & 1.135541 & 1.135747 & 1.135689 & 1.135703 & 1.135638 \\
\hline
\end{tabular}

In the first Scenario (CBR_UBR), the adaptation layer and services in the Data Station for Ftp were assigned as shown in table 11: A lower Upload Response Time and for that matter a better response time was recorded in this scenario for Ftp file such as video because, AAL2 which is good for audio, takes care of the audio aspect of the Ftp file while AAL5 which is good for data files takes care of the data aspect of the Ftp file. It also made use of the Service Class of Voice (UBR) and that of Ftp (UBR) thereby lowering the time spent in downloading the Ftp file.

Table 11: FTP Upload Response Time for scenario 1

\begin{tabular}{|l|l|l|l}
\hline APPLICATION & ATM ADAPTATION LAYER & SERVICE CLASS & OUTPUT \\
\hline Voice & AAL2 & UBR & \multirow{2}{*}{$\mathbf{1 . 1 2 9 1 0 3}$} \\
\hline Ftp & AAL5 & UBR & \\
\hline Email & AAL5 & UBR & \\
\hline
\end{tabular}

In the second Scenario (UBR_UBR), the adaptation layer and services in the Data Station for Ftp were assigned as shown in table 12: A higher Ftp Upload Response Time and for that matter poor response time was recorded because, AAL5 adaptation layer was assigned to the voice aspect of the Ftp file though the data aspect of the Ftp file took advantage of the adaptation layer of Email (AAL5) and its own. It could be deduced that the voice aspect of the Ftp file was delayed which in turn affected the overall Upload response time for the Ftp File. It can therefore be deduced that, in downloading an Ftp File, two different adaptation layers (AAL2 and AAL5) are required, AAL2 to transport the voice aspect and AAL5 to transport the data aspect. It can also be inferred that UBR as a Service Class is better in Uploading Ftp Files. Figure 4 shows a plot of the bit rate against runtime for the two scenarios.

From figure 4 below, it can be seen that, the UBR_UBR scenarios produces a constant response time of 1.135 seconds for all data packets whiles it ranges from 1.115 seconds for low traffic to 1.13 seconds for medium traffic and then drops to 1.125 seconds for higher traffic.
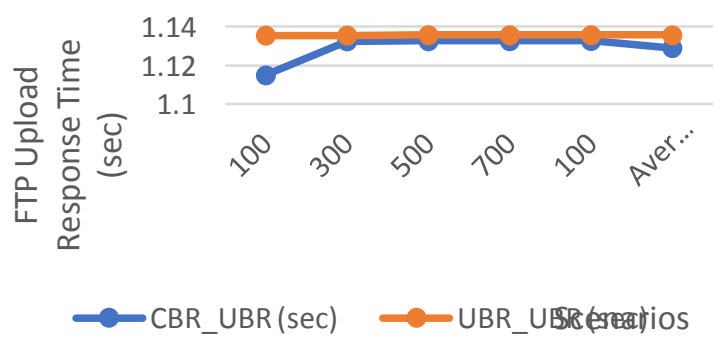

Figure 4: FTP Upload Response time 
5.5 Results for Voice Packet Delay Variation (Sec) An average value of 4.92E-06 seconds and 9.64E-05 seconds were recorded respectively for CBR_UBR and UBR_UBR scenarios. The Variance among end-to-end delays for voice packets in the first scenario is lower than that of the second Scenario shown table12

Table 12: Voice Packet Delay Variation (sec)

\begin{tabular}{|l|c|c|c|c|c|c|}
\hline \multirow{2}{*}{ SCENARIOS } & \multicolumn{5}{|c|}{ VALUES PER STATISTIC } & \multirow{2}{*}{ AVERAGE } \\
\cline { 2 - 6 } & 100 & 300 & 500 & 700 & 1000 & \\
\hline $\begin{array}{l}\text { CBR_UBR } \\
\text { (Sec) }\end{array}$ & $4.89321 \mathrm{E}-06$ & $5.1162 \mathrm{E}-06$ & $4.51736 \mathrm{E}-06$ & $5.1084 \mathrm{E}-06$ & $4.94822 \mathrm{E}-06$ & $4.92 \mathrm{E}-06$ \\
\hline $\begin{array}{l}\text { UBR_UBR } \\
\text { (Sec) }\end{array}$ & $9.4 \mathrm{E}-05$ & $9.62 \mathrm{E}-05$ & $9.73 \mathrm{E}-05$ & $9.72 \mathrm{E}-05$ & $9.74 \mathrm{E}-05$ & $9.64 \mathrm{E}-05$ \\
\hline
\end{tabular}

In the first Scenario (CBR_UBR), the adaptation layer and services in the Voice Station were assigned as seen in table 13: A lower Voice Packet Delay Variation and for that matter a better delay variation was recorded in this scenario because, AAL2 which is good for low bit rate and delay sensitive applications such as voice was used. It also made use of Constant Bitrate (CBR) as the Service Class which is good for applications that requires an unvarying amount of bandwidth and delay sensitive, such as voice.

Table 13: Voice Packet Delay Variation for scenario 1

\begin{tabular}{|l|l|l|l|}
\hline $\begin{array}{l}\text { APPLICATIO } \\
\mathrm{N}\end{array}$ & $\begin{array}{l}\text { ATM } \\
\text { ADAPTATION } \\
\text { LAYER }\end{array}$ & $\begin{array}{l}\text { SERVICE } \\
\text { CLASS }\end{array}$ & OUTPUT \\
\cline { 1 - 2 } Voice & AAL2 & CBR & \multirow{2}{*}{ 4.92E-06 } \\
\cline { 1 - 3 } Ftp & AAL5 & UBR & \\
\hline Email & AAL5 & UBR & \\
\hline
\end{tabular}

In the second Scenario (UBR_UBR), the adaptation layer and services in the Voice Station were assigned as shown in table 14: A higher Voice Delay Variation and for that matter poor time variation was recorded because, AAL5 adaptation layer was assigned to the voice application. AAL5 as an adaptation layer was designed to transport variable-length packets unlike voice. UBR service class was assigned to the voice application. Voice which is a real time application do not require a non-real-time service category such as UBR.

From figure 5, it can be seen that the CBR_UBR scenarios recorded a constant low response of $0.00 \mathrm{E}-100$ seconds for all traffic simulated whiles a higher value of 1.00E.04 seconds for UBR_UBR for all traffic.

It can therefore be deduced that for fast transmission and better quality of sound (voice), AAL2 as an adaptation layer in conjunction with CBR as a service class should be used

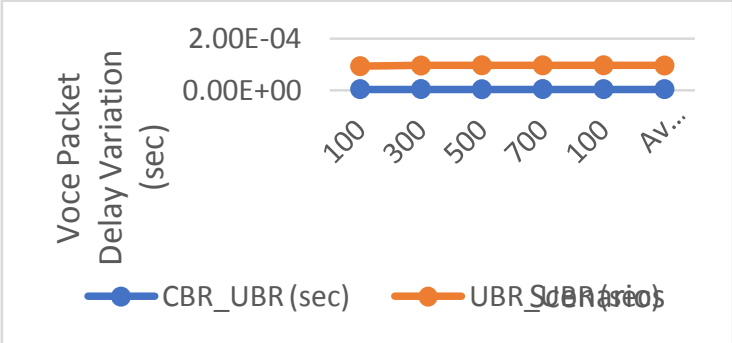

Figure 5: Voice Packet Delay Variation

\subsection{Results for Voice Packet End to End Delay (Sec)}

An average value of 1.82E-01 seconds and 0.1878504 seconds were recorded respectively for CBR_UBR and UBR_UBR scenarios shown in the table 14 .

The total voice packet delay ("analog-to-analog" or "mouthto-ear") for voice packets in the first scenario is lower than that of the second Scenario.

Table 14: Voice Packet End to End Delay (Sec)

\begin{tabular}{|l|c|c|c|c|c|c|}
\hline \multirow{2}{*}{ SCENARIOS } & \multicolumn{5}{|c|}{ VALUES PER STATISTIC } & \multirow{2}{*}{ AVERAGE } \\
\cline { 2 - 6 } & 100 & 300 & 500 & 700 & 1000 & \\
\hline $\begin{array}{l}\text { CBR_UBR } \\
\text { (Sec) }\end{array}$ & 0.181757 & 0.181472 & 0.181642 & 0.181555 & 0.181629 & $1.82 \mathrm{E}-01$ \\
\hline $\begin{array}{l}\text { UBR_UBR } \\
\text { (Sec) }\end{array}$ & 0.187777 & 0.187831 & 0.187859 & 0.187864 & 0.187921 & 0.1878504 \\
\hline
\end{tabular}

In the first Scenario (CBR_UBR), the adaptation layer and services in the Voice Station were assigned as follows in table 15: A lower Voice Packet End to End Delay and for that matter a better delay was recorded in this scenario because, AAL2 which is good for low bit rate and delay sensitive applications such as voice was used. It also made use of Constant Bitrate (CBR) as the Service Class which is good for applications that requires an unvarying amount of bandwidth and are delay sensitive, such as voice.

Table 15: Voice Packet End to End Delay (Sec) for scenario 1

\begin{tabular}{|l|l|l|l|}
\hline APPLICATION & $\begin{array}{l}\text { ATM } \\
\text { ADAPTATION } \\
\text { LAYER }\end{array}$ & $\begin{array}{l}\text { SERVICE } \\
\text { CLASS }\end{array}$ & OUTPUT \\
\hline Voice & AAL2 & CBR & \multirow{2}{*}{$1.82 \mathrm{E}-01$} \\
\hline Ftp & AAL5 & UBR & \\
\hline Email & AAL5 & UBR & \\
\hline
\end{tabular}

In the second Scenario (UBR_UBR), the adaptation layer and services in the Voice Station were assigned as follows in table 16: A higher Voice Packet End to End Delay and for that matter poor time was recorded because, AAL5 adaptation layer was assigned to the voice application. AAL5 as an adaptation layer was designed to transport variable-length packets unlike voice. UBR service class was assigned to the voice application. Voice which is a real time application do not require a non-real-time service category such as UBR.

It can therefore be concluded that to reduce the delay of the 
transmission of voice from End to End, AAL2 as an adaptation layer in conjunction with CBR as a service class should be used.

Table 16: Voice Packet End to End Delay (Sec) For Scenario2

\begin{tabular}{|l|l|l|l|}
\hline APPLICATION & $\begin{array}{l}\text { ATM } \\
\text { ADAPTATION } \\
\text { LAYER }\end{array}$ & $\begin{array}{l}\text { SERVICE } \\
\text { CLASS }\end{array}$ & OUTPUT \\
\hline Voice & AAL5 & UBR & \multirow{2}{*}{0.1878504} \\
\cline { 1 - 3 } Ftp & AAL5 & UBR & \\
\cline { 1 - 3 } Email & AAL5 & UBR & \\
\hline
\end{tabular}

The results for the two scenarios are shown in the figure 7 . UBR_UBR has a high response of 0.188 seconds for low, medium and higher traffic against a low response of 0.080seconds for the three traffic of data packet in CBR_UBR scenarios.

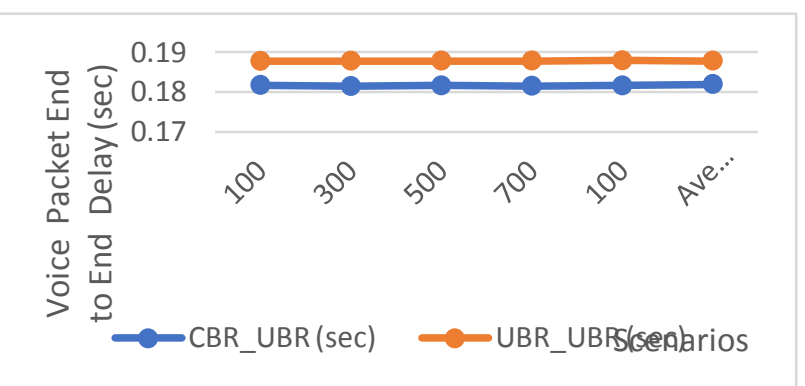

Figure 7: Voice Packet End to End Delay

\section{RELATED REVIEW}

ATM is a technology defined by protocols standardized by the ITU-T, ANSI, ETSI and the ATM forum. ATM is a cell-based switching and multiplexing technology designed for general purpose, connection-oriented transfer mode for a wide range of services [1,3]. The abbreviated header of ATM and fixed length facilitate hardware implementation that result in low delay and high speed [2]. According to [4,5], ATM can be considered to represent a unifying technology because it was designed to transport voice, data, and video (including graphics images) on both local and wide area networks. ATM is universally accepted as the transfer mode of choice for BISDN [6].

The physical layer is divided into two sub-layers. These are physical medium dependent sub-layer and transmission convergence sub-layer. According to [8], the physical layer defines the bit timing and other characteristics for encoding and decoding the data into suitable electrical/optical waveforms for transmission and reception on the specific physical media used

The ATM layer represents the physical interface between the ATM Adaptation layer (AAL) and the Physical layer. When receiving cells from the Physical layer, the ATM layer performs a reverse operation, extracting the five-byte header from each cell [5].

AAL2 is an adaptation layer that is used to multiplex more than one constant low bit rate user information stream on a single ATM virtual connection [9]. This AAL provides for bandwidth efficient transmission of low-rate, short, and variable length packets in delay sensitive applications.

The Constant Bit Rate (CBR) category is intended for applications requiring tightly constrained delay and delay variation. CBR services use ATM Adaptation Layer Type1,[7] because it receives/delivers SDU (Service Data Unit) with a constant bit rate from/to the layer above. The CBR class of service is the preferred choice for many video dial tone service providers [10].

The Variable Bit Rate (VBR) category is intended for applications that shares the requirements for tightly constrained delay and delay variation of CBR traffic, but which transmit at a variable rate. Compressed voice with silence suppression, and variable rate video codecs are examples of this category of traffic. ATM Adaptation Layer Type-2 is proposed for VBR services with a timing relation between source and destination [7], for example VBR Voice or video.

\section{CONCLUSION, RECOMMENDATIONS AND FINDINGS}

From the results of the simulation exercise, it can be conveniently inferred that the choice of an ATM Adaptation Layer type and ATM Service Class has an effect on the performance of a network with respect to the application it carries.

From the conclusion, we recommend that all Information Technology related firms especially Multimedia institutions which make use Voice, Video and busty data, should take into consideration the type of ATM Adaptation Layer and Service Class they chose to optimize the performance of the network during the data's transmission.

To reduce the complex nature of the architecture of ATM Adaptation layers and that of the Service Class, we recommend that a one-all-inclusive Adaptation Layer and Service Class should be developed to dynamically transport all forms of applications be it Voice, Video or Data

\section{REFERENCES}

[1] Ahamed, D. R. (2005-2008). The Role of ATM Technology in Future Data Communication Systems. Journal of Theoretical and Applied Information Technology, 624-628.

[2] Alberto Leon-Garcia and Indra Widjaja. (2002). Communication Networks: Fundamental Concepts and Key Architectures (5th Edition ed.). Tata McGraw-HilL.

[3] David Mc Dysan and Dave Paw. (2003). Communications Networking: ATM and MPLS and Theory and Application. Dreamtech Press.

[4] Held, G. (1999). Understanding Data Communications (6th Edition ed.). USA: New Riders Publishing.

[5] [5] Held, G. (1999). Understanding Data Communications. New Riders Publishing. IEC Tutorial. (2005). Retrieved from ATM Fundamentals: http://www.iec.org/online/tutorials/

[6] R. Guerin, H. Ahmadi and M. Naghshineh. (19991, September 7). Equivalent Capacity and its Application to Bandwidth Allocation in High-Speed Network. IEEE Journal, 968-981.

[7] R. Handel, M. N. (1994). ATM Networks: Concepts, 
Protocols, Applications (2nd Edition ed.). Addison wesly.

[8] S. K. Rao and M. Hatamian. (1995, April). The ATM Physical Layer. ACM SIGCOMM Computer Communication Review, 73-81.

[9] Schicker, P. (1997, February 2). B-ISDN ATM Adaptation Layer Type 2 Specification. ITU-T
Recommendation, 1-363.

[10] Sudhir Dixit and Paul Skelly. (1995, October). MPEG-2 over ATM for Video Dial Tone Networks: Issues and strategies. IEEE Network, 30-40.

[11] Suzuki, T. (1994, April). ATM Adaptation Layer Protocol. IEEE Communications Magazine, 80-83. 